\title{
Queer inclusive planning \\ Raumansprüche und queeres Selbstverständnis in einer heteronormativen \\ Gesellschaft
}

Rogério de Jesus Pereira Lopes

\section{Einleitung}

Während auf politischer und gesellschaftlicher Ebene in zunehmendem Umfang darüber diskutiert wird, inwieweit Minderheiten gegenüber der Mehrheitsgesellschaft gleichgestellt und geschützt werden sollen (vgl. de Tomás 2016, Horchler 2016, Scally 2015), setzt sich insbesondere die Stadtplanung nur unzureichend mit den Bedürfnissen verschiedener sozialer Gruppen auseinander (vgl. Fincher/Iveson 2008: 1f.). Dies ist hinsichtlich ihrer Funktion als „conscious effort to direct social processes to attain goals" (Fainstein/Fainstein 1996: 256) kritisch zu hinterfragen. So ist davon auszugehen, dass verschiedene planerische Maßnahmen und Strategien sowie deren Implementierung einen wesentlichen Einfluss auf soziale Praktiken und das Miteinander in der Gesellschaft haben (vgl. Fainstein/ Fainstein 1996: 256).

Trotz oder gerade wegen der unzureichenden Auseinandersetzung mit den Bedürfnissen unterschiedlicher sozialer Gruppen, ist im Laufe der Jahre eine zunehmende Einbindung partizipatorischer Planungsmaßnahmen zu erkennen (vgl. Diebäcker 2008: 279f.). Diese stehen im Zusammengang mit dem communicative turn. Damit wird die Veränderung des Planungsverständnisses von einem technokratisch organisierten und top-down orientierten Prozess zu einem flexibleren, bottom-up- und konsensbasierten Prozess beschrieben (vgl. Kühn 2008: 231f.). So haben vor allem Beteiligungsprozesse auf Quartiers- und Stadtebene derzeit eine immer größer werdende Bedeutung.

Der beschriebene Paradigmenwechsel in Bezug auf die Einbindung von Bedürfnissen der Stadtgesellschaft sowie die zunehmende Bedeutung von Diversität in heutigen Gesellschaften klammern jedoch die Bedürfnisse und Ansprüche geschlechtlicher und sexueller Minderheiten aus (vgl. Doan 2011a: 1ff.). Dies hat möglicherweise Folgen für das Selbstverständnis und Selbstbewusstsein queerer Bürger_innen, da eine Ausklammerung von Bedürfnissen sozialer Minderheiten zu einer Herabwürdigung ihrer Identitäten führt (vgl. Schulz 2009: 18f.). In Bezug auf die Rolle von Queers in der 
Stadtplanung möchte ich deshalb in diesem Essay diskutieren, welche Raumansprüche Queers haben und inwiefern ein queer inclusive planning Ansatz in der Stadtplanung weiterentwickelt werden sollte. Nach einer Definition des Begriffes ,queer ' werden die Ziele des queer inclusive planning Ansatzes dargelegt, um in einem weiteren Abschnitt exemplarische Raumansprüche von Queers vorzustellen. Im letzten Teil des Textes werden die Potentiale und Herausforderungen eines queer inclusive planning Ansatzes zusammengefasst und diskutiert.

\section{Queers in einer heteronormativen Gesellschaft}

Unter dem Label Queers versammeln sich Menschen sehr unterschiedlicher Lebensrealitäten (vgl. Jagose 1996: 17f.). Gemeinhin bezeichnen sich Menschen als queer, die Kritik an der heteronormativ und zweigeschlechtlich ausgerichteten Gesellschaft üben und beziehungsweise oder sich selbst in diesem Spektrum nicht wiederfinden. Hierbei wird Heteronormativität als Gesellschaftsmodell verstanden, welches „Heterosexualität als Norm der Gesellschaftsverhältnisse [...] [sowie] symbolische Ordnung [begreift] und das Gefüge der gesellschaftlichen Organisation strukturiert“ (Wagenknecht 2007: 17). Ebenso wird von lediglich zwei möglichen Geschlechtszuordnungen ausgegangen (vgl. ebd.). Insbesondere definieren sich solche Individuen als queer, die nicht (ausschließlich) heterosexuell leben und beziehungsweise oder nicht in das zweigeschlechtliche Schema der Gesellschaft einzuordnen sind oder eingeordnet werden wollen. Wenngleich in den meisten Fällen von Lesben, Schwulen, Transidenten, intersexuellen Menschen sowie Bi- und Pansexuellen die Rede ist, zeichnet sich der Begriff Queer durch seinen dynamischen Charakter aus. Es gibt keine feste Definition von queer. Vielmehr bietet der Terminus Raum für Neuinterpretationen und suggeriert einen offenen Zugang für all diejenigen, die mit ihrer Lebensweise den Wertevorstellungen der gesellschaftlich dominanten Ordnung widersprechen (vgl. Czollek/Perko/Weinbach 2009: 33, Jagose 1996: 1ff.).

Die Selbstbezeichnung und -zuschreibung queer steht in engem Zusammenhang mit den in den 1990er-Jahren aufkommenden queer politics und der daraus resultierenden queer theory. Das Hauptanliegen der Vertreter_innen dieser Theorie besteht darin, ein Gegenkonzept zum vorherrschenden heterosexuell und zweigeschlechtlich geprägten Verständnis der Gesellschaft herzustellen, welches die Grenzen von Identifikation und Begehren zwischen den sozialen Gruppen überschreitet (vgl. Jagose 1996: 17f.). Hierbei werden bestehende Annahmen und Normen der Mehrheitsgesellschaft hinsichtlich ihres ausschließenden Charakters hinterfragt und dekonstruiert. Dieser Prozess der Dekonstruktion, welcher im Bereich der queer theory als queering bezeichnet wird, bildet eine Grundlage, um bestehende Normen zu „ent-selbstverständlichen [sowie zu] ent-naturalisieren“(Klapeer 2015: 30).

Infolge der offenen Definition der Selbstbezeichnung queer ist davon auszugehen, dass Queers unterschiedliche Lebensrealitäten aufweisen, denen im Rahmen einer Analyse queerer Raumansprüche Rechnung getragen werden muss. Ein Beispiel hierfür bilden die Unterschiede zwischen Lesben und Schwulen. Homosexuelle Männer verfügen gegenüber homosexuellen Frauen gemeinhin über eklatante finanzielle Vorteile, die oft mit einer 
größeren Sichtbarkeit schwulen Lebens einhergehen. Es wird argumentiert, dass die divergente - und paradoxerweise heteronormative - Sozialisierung von Männern und Frauen einen wesentlichen Einfluss hierauf habe (vgl. Castells 1983: 140). Männer und Frauen werden aus heteronormativer Sicht als Gegenpole betrachtet. Diese Geschlechterbinärität bildet die Grundlage für Herrschaftskategorien und Unterdrückungsverhältnisse, die zu der privilegierten Position von Männern gegenüber Frauen führt. Gleichzeitig begünstige dieses ungleiche Machtverhältnis, so Antke Engel, den gesellschaftlichen Ausschluss von Individuen, die sich zwischen oder außerhalb der beiden Geschlechtskategorien befinden (1994: 3f.). Angelehnt an die Abwertung von Frauen erfahren Lesben, im Vergleich zu Schwulen, nicht nur Diskriminierung aufgrund ihrer sexuellen Orientierung, sondern auch wegen ihrer Geschlechtszuordnung. Angesichts dieser Mehrfachdiskriminierung definieren sich viele Lesben, im Vergleich zu Schwulen, über gegenkulturelle oder feministische Gruppierungen und weniger über ihre sexuelle Orientierung (vgl. Frisch 2002: 257, Forsyth 2001: 346). Ebenso bestehen Unterschiede zwischen homosexuellen und transidenten Queers in Bezug auf ihre Sichtbarkeit. Der Begriff Transident fasst verschiedene Individuen zusammen, die sich temporär oder dauerhaft außerhalb der binären Geschlechterordnung positionieren. So werden Trans_frauen und Trans_männer, aber auch dragkings, dragqueens, Crossdresser_innen und andere Individuen miteinbezogen (vgl. Doan 2007: 59). Trotz ähnlicher Rahmenbedingungen nehmen viele Lesben und Schwule die Bedürfnisse von Transidenten nur unzureichend wahr, sodass von einer bedingten Akzeptanz auszugehen ist. Insbesondere die Anpassung der Lebensformen an heterosexuelle und zweigeschlechtlich organisierte Normen unter Lesben und Schwulen hat einen enormen Einfluss hierauf. Dies trägt einerseits zur Verfestigung von Zweigeschlechtlichkeit unter Queers bei. Andererseits marginalisiert es all diejenigen, die sich außerhalb der traditionellen Geschlechterrollen befinden (vgl. ebd.: 62f.).

Anhand der beschriebenen Unterschiede wird deutlich, dass queere Bürger_innen nicht als homogene, soziale Gruppe angesehen werden können. Vielmehr lassen sich unter ihnen Machtverhältnisse erkennen, die zu unterschiedlichen Lebensrealitäten führen. Darüber hinaus lassen sich diese Unterschiede zwischen queeren Individuen auch hinsichtlich der sozioökonomischen und ethnischen Herkunft erkennen, auf die im späteren Verlauf des Essays in Teilen näher eingegangen wird. Insofern ist davon auszugehen, dass allein die heterogene,Gruppe von Queers unterschiedliche Erwartungen und Ansprüche an den städtischen Raum hat.

\section{Queer inclusive planning als Reaktion auf eine heteronormativ organisierte Stadtplanung}

Ausgehend von der Annahme, dass die Lebensrealitäten und Bedürfnisse geschlechtlicher und sexueller Minderheiten in der Stadtplanung keine Relevanz haben, argumentieren US-amerikanische Wissenschaftler_innen, dass ein inklusiverer Ansatz verfolgt werden sollte, der queere Bedürfnisse miteinbezieht. Diese Forderung orientiert sich insbesondere an den gewonnenen Erkenntnissen über den exkludierenden und heteronormativen Charakter der 
Stadtplanung und dessen Auswirkungen auf Queers des US-amerikanischen Stadtplaners Michael Frisch (vgl. 2002: 263f., Doan 2011a: 1ff.; 2015: 7ff., Forsyth / Cheung 2001) Die Stadtplanung sei, so Frisch, in ihrer bisherigen Form ein durch dichotome Denkstrukturen geprägtes Instrument, welches etwa Ordnung und Unordnung, Öffentlichkeit und Privatheit sowie Produktion und Reproduktion gegenüberstelle. Diese Gegenüberstellungen führten weiterhin zu Macht- sowie Unterdrückungsverhältnissen, die mit den Annahmen der Heteronormativität und Binarität der Geschlechter zusammenhingen (vgl. Frisch 2002: 254ff.).

Ordnung sei laut Frisch darüber hinaus in den USA - insbesondere seit den 1950er-Jahren - über die Schaffung suburbaner Räumen hergestellt worden, in denen vor allem heterosexuelle Familien angesiedelt worden wären. Im Vergleich dazu würden innerstädtische Standorte mit Unordnung assoziiert und seien vor allem von alleinstehenden Personen sowie kinderlosen Paaren bewohnt. Aufgrund der Tatsache, dass Frauen- und Männerpaare zum Zeitpunkt der Analyse Frischs nicht als Familie anerkannt wurden und kein Anrecht auf Adoption hatten, sieht er hier einen Zusammenhang zu den Macht- und Unterdrückungsverhältnissen zwischen Heterosexuellen und Homosexuellen. Wo Unordnung herrsche, versuche die Stadtplanung seiner Meinung nach Wege zu finden, um Ordnung zu schaffen. Insofern sei davon auszugehen, dass Heterosexualität gegenüber Homosexualität privilegiert werde (vgl. Frisch 2002: 259f.).

Hierauf aufbauend geht Frisch davon aus, dass Stadtplanung ein ,heterosexist project“ (2002: 254) sei, welches in vielerlei Hinsicht all diejenigen diskriminiere, die der heteronormativen Mehrheitsgesellschaft widersprächen (vgl. ebd.: 254ff.). Gleichwohl argumentiert die Stadt- und Regionalplanerin Petra Doan, dass Stadtplanung aufgrund ihres gesamtgesellschaftlichen Auftrags und dem hiermit verbundenen öffentlichen Interesse nicht in der Lage sei, auf Bedürfnisse einzelner Individuen einzugehen. Durch ihre mehrheits- und konsensorientierte Ausrichtung sei Stadtplanung stets darum bemüht, normative Annahmen zu verfolgen und zu verfestigen. Demnach stünden individuelle Bedürfnisse einzelner Minderheiten stets im Konflikt zu dominierenden Vorstellungen der Mehrheitsgesellschaft (vgl. 2011a: 3ff.).

Ein queere Bedürfnisse einbindendes Planungskonzept könnte diese ausschließenden Strukturen überwinden. So könnte ein Ansatz des „queer inclusive planning" (Frisch 2002: 264) queere Anforderungen und Ansprüche an den Raum in die Stadtplanung integrieren (vgl. ebd.). Ein inklusiver Ansatz wie dieser ermöglichte folglich eine kritische Auseinandersetzung mit der vorherrschenden Stadtplanungspraxis und deren Auswirkungen auf marginalisierte Individuen (vgl. Klapeer 2015: 29f.).

\section{Queere Raumansprüche in einer heteronormativen Gesellschaft}

Ausgehend von der Annahme, dass Stadtplanung heteronormative Ordnungsmuster widerspiegele, muss davon ausgegangen werden, dass die Bedürfnisse und Raumansprüche queerer Individuen weitgehend unbeachtet bleiben. Über die spezifischen Raumansprüche von Queers gibt es jedoch nur wenige Erkenntnisse aus der Forschung. Um mehr über diese 
Raumbedürfnisse zu erfahren, habe ich im Rahmen meiner Bachelorarbeit Interviews in Dortmund durchgeführt. So sprach ich mit der Vertreterin eines Transidentenvereins, mit einem Mitarbeiter des Stadtplanungsund Bauordnungsamtes und der Koordinierungsbeauftragten für Lesben, Schwule und Transidente der Stadt Dortmund sowie mit dem Vorsitzenden eines Lesben- und Schwulenvereins. Ich orientierte mich in meiner Arbeit an den Ergebnissen angelsächsischer Analysen, in denen queere, insbesondere schwule Ansprüche an den städtischen Raum und an die Stadtplanung thematisiert wurden (vgl. Castells 1983, Doan 2011a; 2011b; 2011c; 2015, Doan/Higgins 2011, Dubrow 2011, Forsyth 2001; 2011, Hubbard 2012, van Liehout 1997, Frisch 2002). Die Interviews dienten dazu, die in diesen Studien ausgearbeiteten Erkenntnisse zu vertiefen, sie hinsichtlich ihrer Übertragbarkeit auf den deutschen Kontext zu analysieren und ihre Relevanz für Queers in Dortmund zu untersuchen. Aufgrund der unterschiedlichen Lebenssituationen queerer Individuen, sollten weniger allgemeine Ansprüche zusammenzutragen werden. Vielmehr sollten anhand von Beispielen Erkenntnisse darüber gewonnen werden, inwiefern die bisherige Stadtplanungspraxis die Bedürfnisse von Queers einschränkt.

In der Analyse der Interviews habe ich drei exemplarische Raumansprüche von Queers herausgearbeitet, die durch die Stadtplanung eingeschränkt werden. Zum einen konnte ich feststellen, dass sowohl die Sicherheit im öffentlichen Raum als auch die Sichtbarkeit queeren Lebens eine große Bedeutung für Queers haben. Zum anderen spielt die Wahrung von Cruisingstandorten insbesondere für schwule und bisexuelle Männer eine große Rolle. Aufgrund der Dominanz von Literatur über weiße Schwule - gegenüber anderen geschlechtlichen und sexuellen Minoritäten - beziehen sich die ausgearbeiteten Bedürfnisse und Ansprüche mehrheitlich auf diese Gruppierung.

\section{Sicherheit im öffentlichen Raum}

Als erster Raumanspruch konnte Sicherheit im öffentlichen Raum identifiziert werden (vgl. de Jesus Pereira Lopes 2015: 20). Trotz zunehmender Toleranz gegenüber Queers, sind diese nach wie vor zahlreichen Anfeindungen im öffentlichen Raum ausgesetzt (vgl. Forsyth 2011: 23). Vor allem Trans_frauen of color sind überdurchschnittlich oft Opfer von gewalttätigen Angriffen. Dies ist auf die Mehrfachdiskriminierung hinsichtlich der geschlechtlichen Identität, der Hautfarbe sowie der Geschlechtszuweisung als Frau zurückzuführen und trägt dazu bei, dass Trans_frauen im Allgemeinen sowie Trans_frauen of color im Speziellen weniger Zeit im öffentlichen Raum verbringen (vgl. National Coalition of Anti-Violence Programs 2015: 8ff.). Vergleicht man deren Situation mit der von Lesben oder Schwulen, fällt auf, dass diese Gruppen die Gefahren gewalttätiger Übergriffe teilweise durch straight acting reduzieren. Hierbei handelt es sich um eine Anpassung des Verhaltens und des geschlechtsbezogenen Auftretens an die Erwartungen der Mehrheitsgesellschaft. Dies ermöglicht es vielen Lesben und Schwulen, unerkannt zu bleiben (vgl. Hubbard 2012: 105). Jedoch ist es fraglich, ob eine solche Anpassung nicht zu einer Einschränkung des Selbstverständnisses von Queers und zur weiteren Marginalisierung sexueller und insbesondere geschlechtlicher Minderheiten führt. 
Gewalttätige Übergriffe und straight acting zeigen, dass sichere öffentliche Räume eine besondere Funktion für ein selbstbestimmtes und selbstbewusstes Leben haben können. Insofern müssen die gegenwärtigen Maßnahmen zur Förderung der Sicherheit von öffentlichen Räumen infrage gestellt werden. Insbesondere stellt sich die Frage, ob solche Konzepte tatsächlich zu einer größeren Sicherheit für alle Bürger_innen oder vielmehr zu einer Verfestigung heteronormativer Denkmuster führen.

In den USA orientieren sich Umgestaltungsmaßnahmen zur Erhöhung der Sicherheit im öffentlichen Raum oftmals an den Prinzipien des defensible space. Diese führten zwar sichtlich zu einer Reduktion von Gewalttaten, allerdings lägen diesen Prinzipien - laut Doan - normative Annahmen zu Grunde, die im Konflikt mit den Bedürfnissen transidenter Bürger_innen stünden (vgl. 2011b: 98). Insbesondere veränderte Designs von öffentlichen Räumen sowie der Einsatz von Nachbarschaftswachen im Sinne einer gemeinschaftlichen Überwachung werden hierbei als Problem erkannt. Denn für viele Transidente ist es wichtig, sich anonym im öffentlichen Raum bewegen zu können. Eine offenere Gestaltung zuvor unsicherer öffentlicher Räume führe aus Doans Sicht aber dazu, dass Transidente leichter erkannt und stärkeren Repressalien ausgesetzt seien. Dies habe letztlich zur Folge, dass Transidente diese Orte seltener aufsuchten oder gänzlich mieden (vgl. ebd.). So bieten beispielsweise Einfriedungen - etwa in Form von Hecken - Transidenten die Möglichkeit, sich vor abwertenden Blicken anderer Individuen oder vor sonstigen Diskriminierungen zu schützen. Nichtsdestotrotz werden gerade in Angsträumen Hecken zurückgeschnitten, um das allgemeine Sicherheitsgefühl zu steigern.

Neben der Gestaltung von öffentlichen Plätzen und Parks spielt insbesondere die Planung von Sanitäranlagen eine große Rolle bei der Erhöhung des Sicherheitsempfindens für Transidente. Im Rahmen einer Analyse zur Errichtung von genderneutralen Sanitäranlagen in Seattle wurde festgestellt, dass Trans_frauen sowie Trans_männer in konventionellen Anlagen in hohem Maße Bedrohungen und anderen Arten der Diskriminierung ausgesetzt sind. Die Gefahr der Anfeindung werde laut Young durch semiprivate Bereiche innerhalb der Anlagen in Form von Zwischenräumen für Waschbecken oder Urinale verstärkt (vgl. 2016: 10ff.). Auf Grundlage dieser Ergebnisse werde eine Abkehr von geschlechtertrennenden Sanitäranlagen empfohlen. Ebenso könne die Planung von einzelnen Toilettenräumen mit individuellen Zugängen und ohne Zwischenräume für Waschbecken oder Urinale zu einem gesteigerten Sicherheitsempfinden beitragen. Demnach solle jeder Toilettenraum mit einem Urinal ausgestattet und die Waschbecken in Form von Sammelstellen vor den Sanitäranlagen platziert werden. Durch die Auslagerung der Waschbecken in den öffentlichen Raum würde das Potential der sozialen Kontrolle gesteigert (vgl. ebd.: 21ff.).

Die angeführten Beispiele sowie die Kritik Petra Doans zeigen, inwieweit der Großteil bisheriger Sicherheitskonzepte die Lebensrealitäten geschlechtlicher und sexueller Minoritäten ausklammert und zu einem stärkeren Rückzug ebendieser Individuen aus dem öffentlichen Raum führt. Insofern benötigt ein Sicherheitskonzept für öffentliche Räume, welches einen queer inclusive planning Ansatz verfolgt, eine Einbindung verschiedener Lebensrealitäten, um queeren Bürger_innen einen sicheren Aufenthalt zu ermöglichen. 


\section{Sichtbarkeit queerer Identität und queeren Lebens im Stadtraum}

Die Sichtbarkeit queerer Identität und queeren Lebens im Stadtraum ist ein weiterer Raumanspruch, der für die Emanzipation geschlechtlicher und sexueller Minderheiten eine besondere Bedeutung hat (vgl. de Jesus Pereira Lopes 2015: 22). Im Vergleich zur Mehrheitsgesellschaft und deren Errungenschaften im Laufe der Geschichte, erfahren Queers nur wenig Beachtung im städtischen Raum. Dies fällt insbesondere im Bereich der Denkmalerhebung auf, da in diesem Bereich die queere Geschichte eine sehr untergeordnete Rolle spielt. Deshalb fordert Architektin Gail Dubrow beispielsweise solche Orte verstärkt in den Mittelpunkt zu stellen, die im Rahmen der Bürger_innenrechtsbewegungen der 1970er-Jahre eine besondere Rolle spielten oder an denen sich Queers in der Vergangenheit politisch und gesellschaftlich organisierten (vgl. 2011: 55ff.).

Ein internationales Beispiel für Maßnahmen zur stärkeren Sichtbarkeit und Betonung queerer Geschichte und Emanzipation ist die Erhebung des Stonewall Inn und des angrenzenden Parks in New York zu nationalen Denkmälern der USA. Im Jahr 1969 wehrten sich an diesen Orten Queers gegen Polizeirazzien, deren Ziel es war, Individuen der queeren Community festzunehmen. Durch ihren Widerstand unterstrichen sie ihren Anspruch auf Gleichberechtigung und Anerkennung gegenüber der Mehrheitsgesellschaft. Diesen Aufständen folgten landes- und weltweite Demonstrationen (vgl. Associated Press 2015, National Park Service 2016).

Auch Deutschland könnte in Bezug auf die queere Emanzipation als Beispiel interessieren, da die gesellschaftliche Toleranz gegenüber Queers einem stetigen Wandel unterzogen war. Insofern ist davon auszugehen, dass eine große Anzahl an Gebäuden und Orten existiert, die im Laufe der Zeit von Queers genutzt wurden und eine besondere Bedeutung für die Emanzipationsbewegungen hatten. Vor allem in den 1920er-Jahren herrschte in deutschen Großstädten und insbesondere in Berlin ein weitgehend liberaler Umgang mit Queers (vgl. Wortmann 2008: 123), was sich an der großen Anzahl queerer Lokale und Institutionen zeigte (vgl. ebd.: 14). Infolge der Machtergreifung der Nationalsozialist_innen im folgenden Jahrzehnt endete dieser liberale Umgang abrupt (vgl. ebd.: 126). Obwohl sich in den Nachkriegsjahren weitreichende politische und gesellschaftliche Veränderungen vollzogen, wurden Queers weiterhin kriminalisiert und diskreditiert (vgl. Dworek 2012: 46-54). Nichtsdestotrotz, haben die politischen Aufstände und Bürger_innenrechtsbewegungen in der zweiten Hälfte des 20. Jahrhunderts zu einer gesteigerten Toleranz gegenüber Queers beigetragen. Insofern versinnbildlichen diese Bewegungen den Kampf der Queers um Gleichberechtigung innerhalb der Gesellschaft.

Dubrow geht davon aus, dass Maßnahmen zur Sichtbarkeit queeren Lebens dazu beitrügen, die Hegemonie heteronormativer Geschichte und Gesellschaftsstrukturen infrage zu stellen. Neben der Erhebung von historischen Orten queerer Emanzipation könnten bereits bestehende Denkmäler, deren queerer Hintergrund - wie etwa die lesbische Identität einer Künstlerin - aufgrund heteronormativer Machtstrukturen nicht thematisiert werde, hinsichtlich ihres nicht-normativen Hintergrunds dekonstruiert werden 
(vgl. Dubrow 2011: 55ff.). Auch wenn die Sichtbarkeit queerer Identität und queeren Lebens einen Beitrag zu einer höheren Toleranz gegenüber Queers leisten kann, ist zu beachten, dass nicht alle Individuen die Betonung der geschlechtlichen oder sexuellen Identität als bedeutsam ansehen. Ebenso stellt sich die Frage, inwieweit solche Maßnahmen in einer von Männern dominierten Gesellschaft lesbische und transidente Belange sichtbarer machen können. Auch im Bereich der Sichtbarmachung queeren Lebens ist also ein offener Diskurs darüber erforderlich, inwieweit solche Maßnahmen zugleich zu einer stärkeren Sichtbarkeit von Frauen sowie Transidenten beitragen können.

\section{Wahrung von Cruisingstandorten für Schwule}

Als dritter Raumanspruch ist die Wahrung von Cruisingstandorten für Schwule aus der Analyse hervorgegangen (vgl. de Jesus Pereira Lopes 2015: 26). Als Cruising wird eine Form der aktiven Suche nach sexuellen Partner_innen durch eine codierte Körpersprache sowie der anschließende Sex im öffentlichen oder privaten Raum bezeichnet (vgl. Lee 1990: 284f.). Insbesondere unter schwulen Männern wird Cruising als Bestandteil der schwulen Kultur angesehen (vgl. Koordinierungsstelle u. Stadtplanungsamt 9.7.2015). Die besondere Bedeutung von Cruisingstandorten ergibt sich aus der jahrelangen Kriminalisierung von Sex unter Männern in Deutschland und der weiterhin bestehenden gesellschaftlichen Ächtung. So suchen viele schwule Männer gezielt nach Wegen, ihren sexuellen Bedürfnissen unerkannt nachzukommen. Weil sie in der eigenen Wohnung der sozialen Kontrolle durch ihre Mitmenschen unterliegen, weichen viele Männer auf öffentlich zugängliche Orte oder andere private Räume aus. Gleichwohl basiert Cruising insbesondere heutzutage auf dem weitergehenden Wunsch nach Erotik und Sex außerhalb der privaten Räume, welcher sich - infolge der Wahrnehmung des Cruisings als Bestandteil schwuler Kultur - weiter verfestigt hat.

Obwohl oder gerade weil diese Rahmenbedingungen für Schwule bekannt sind, wird in städtischen Räumen auf verschiedene Weise versucht, sexuelle Handlungen unter Männern zu unterbinden. Am Beispiel des Russel Square und Bloomsbury Square in London zeigt sich, wie Cruisende an öffentlich zugänglichen, aber privaten Grünflächen durch Umgestaltungsmaßnahmen verdrängt werden. Der sich in Privatbesitz befindende Bloomsbury Square wurde bereits seit dem 18. Jahrhundert für Cruising genutzt. Der angrenzende Russel Square hingegen wurde vor allem in den 1980er-Jahren zu einem bekannten Ort für sexuelle Handlungen (vgl. Andersson 2012: 1082ff.). Während die Polizei in den 1990er-Jahren einen toleranten Umgang damit pflegte, entschieden die zuständigen Behörden und Eigentümer_innen, den Russel Square in mehreren Schritten umzugestalten. Hierbei wurden zunächst Büsche und Hecken zurückgeschnitten, die den Cruisenden als Rückzugsorte dienten. In den Folgejahren wurde die Grünfläche eingezäunt und fortan über Nacht geschlossen. Als Reaktion hierauf wichen viele Cruisende auf den Bloomsbury Square aus, der allerdings im späteren Verlauf ebenfalls umgestaltet wurde. Hierbei wurde der zentrale Treffpunkt aller Cruisenden durch einen Spielplatz ersetzt (vgl. ebd.: 1087ff.).

Insbesondere die Umwidmung des zentralen Cruisingtreffpunkts kann als Beispiel dafür angeführt werden, wie heteronormative Ideologien und 
Werte die Nutzung des Raums bestimmen und sexuelle Minderheiten verdrängt werden (vgl. Andersson 2012: 1090). Es wird verkannt, dass öffentlich zugängliche Räume Funktionen haben, die über die heteronormativen Deutungen hinausgehen. Da das Cruising als wichtiger Bestandteil schwuler Kultur wahrgenommen wird, sollte im Rahmen eines queer inclusive planning eine Wahrung solcher Standorte angestrebt werden (vgl. de Jesus Pereira Lopes 2015: 28).

Die herausgearbeiteten Raumansprüche von Queers bilden aufgrund des bisher unzureichenden Diskurses über Belange und Ansprüche geschlechtlicher und sexueller Minderheiten an den Raum sowie deren mangelhafte Einbindung in die Planungskonzepte lediglich einen Ausgangspunkt für die Entwicklung eines queer inclusive planning Ansatzes (vgl. de Jesus Pereira Lopes: 47). Dennoch zeigen sie, wie heteronormative Ordnungsmuster und Annahmen nicht-queere Individuen privilegieren und Queers einen selbstbestimmten Zugang zum Stadtraum erschweren. Deutlich zu sehen ist dies am Umgang mit Cruisingstandorten, durch den Frischs Interpretation der Stadtplanung als heterosexistisches Projekt bestätigt wird. Die einer heteronormativen Annahme zugrundeliegende Verortung der Sexualität im Privatem sowie die Privilegierung der Heterosexualität gegenüber der Homosexualität führe zu einer Entwürdigung und zum Ausschluss von Queers (vgl. Frisch 2002: 254ff.). Weil Stadtplanung existierende gesellschaftliche Normen widerspiegelt, verfestigt und bestärkt sie die Marginalisierung all derjenigen, deren Lebensrealität den normativen Annahmen widerspricht.

\section{Herausforderungen und Potentiale eines queer inclusive planning Ansatzes}

Die exemplarischen Raumansprüche von Queers lassen erkennen, inwiefern Stadtplanung und Raum durch normative Annahmen geprägt sind. Ein queer inclusive planning Ansatz würde Queers darin unterstützen, einen selbstverständlicheren Umgang mit der eigenen geschlechtlichen und sexuellen Identität im städtischen Raum zu entwickeln. Darüber hinaus ermöglicht ein solcher Ansatz, Erkenntnisse darüber zu gewinnen, inwiefern Stadtplanung auch andere Minderheiten ausschließt und den Zugang zum städtischen Räumen versperrt.

Die Einbeziehung queerer Belange in die Stadtplanung kann auch einen positiven Beitrag für andere Minderheiten leisten. Queer und critical race theories setzen sich bereits mit dem Problem der Mehrfachdiskriminierung auseinander und prägten den Begriff queer ethnicity und queer of color. Diese umfassen queere Menschen, die von Rassifizierung betroffen sind, die einen Migrationshintergrund aufweisen oder queere Migrant_innen sind. Es wird davon ausgegangen, dass mehrfach diskriminierte Personen auf besondere Weise benachteiligt werden. Mithilfe dieses Ansatzes wird versucht, normative Annahmen hinsichtlich ihrer exkludierenden Strukturen für mehrfach diskriminierte Individuen zu dekonstruieren (vgl. El-Tayeb 2015: 44ff.). In Bezug auf die Stadtplanung ist folglich zu erwarten, dass nicht nur Bedürfnisse und Ansprüche von Queers in öffentlichen Debatten und in der Stadtplanung unbeachtet bleiben. Zahlreiche ethnische, geschlechtliche, sexuelle oder nationale Minderheiten werden im Rahmen der Stadtplanung 
ausgeklammert. Darüber hinaus bleiben insbesondere die Belange mehrfach diskriminierter Individuen unbeachtet. Eine zeitgemäße Stadtplanung sollte auf die Bedürfnisse unterschiedlicher Individuen eingehen, ohne andere soziale Gruppen in ihren Möglichkeiten einzuschränken.

Zwar werden bereits partizipative Maßnahmen zur besseren Einbindung der Bedürfnisse von Bürger_innen ergriffen, diese orientieren sich jedoch an der Mehrheitsgesellschaft. Gesellschaftlich verankerte Machtverhältnisse zwischen unterschiedlichen sozialen Gruppen übertragen sich so auf konsensbasierte Beteiligungsmaßnahmen. Akteur_innen in einer höheren Machtposition hätten hierbei Vorteile gegenüber Minderheiten (vgl. Stock 2008: 2ff.). Darüber hinaus nähmen insbesondere solche Menschen an partizipationsorientierten Planungsprozessen teil, die sich bereits in einer privilegierten Position befänden und erlernt hätten, dass sie etwas bewirken können (vgl. Huning 2014: 36). Ebenso trägt die fehlende Sensibilisierung von Planungsakteur_innen bezüglich der Benachteiligung von geschlechtlichen und sexuellen Minderheiten dazu bei, dass deren Belange keine Berücksichtigung finden. Vielmehr bedarf es Beteiligungsmaßnahmen, die gezielt auf Queers zugeschnitten sind, sich kritisch mit deren Belangen auseinandersetzen und hierbei bestehende Machtverhältnisse zwischen Schwulen, Lesben, Transidenten sowie anderen Queers durchbrechen.

Gleichwohl merkt die Raumplanerin Sandra Huning an, dass zielgruppenorientierte Partizipationsmaßnahmen nicht dazu in der Lage seien, die Belange ganzer Bevölkerungsgruppen aufzufangen. Es müsse in Frage gestellt werden, inwiefern zielgruppenorientierte Maßnahmen eine Form von Fremdzuschreibung seien und hierdurch zugleich „Markierungen des ,Anderen““ (2014: 36) hervorriefen (vgl. ebd.). So könne ein postkategorialer Ansatz, welcher die ausschließenden Prozesse in den Fokus stelle - und weniger die Belange vermeintlich homogener sozialer Gruppen -, Abhilfe leisten. Übertragen auf die Belange geschlechtlicher und sexueller Minderheiten würden Planende demnach nicht nach den Belangen von Queers fragen. Vielmehr würde der Frage nachgegangen werden, wie eine nichtheteronormative Stadt aussähe (vgl. ebd.: 36ff.).

Dieser Vorstellung steht allerdings entgegen, dass Planende nur geringe Kenntnisse darüber besitzen, inwiefern ihre Arbeit heteronormative Annahmen und Muster reproduziert und räumlich verortet. Dies ist im Bereich der Stadtplanung insbesondere an der begrenzten wissenschaftlichen Auseinandersetzung mit der Rolle von Queers zu erkennen (vgl. Doan 2011a: 7ff.). Insofern bedarf die Frage danach, wie eine nicht-heteronormative Stadt aussehen könnte, zunächst grundlegende Kenntnisse darüber, inwiefern die aktuelle stadtplanerische Praxis einen exkludierenden, heteronormativen Charakter aufweist. Hierbei könnte die Frage nach Raumansprüchen von Queers unterstützend wirken, da sie erste Erkenntnisse darüber liefern kann, inwieweit die Stadtplanung zur Einschränkung ebendieser Ansprüche führt. Darüber hinaus können die erarbeiteten Raumansprüche in einem nächsten Schritt dabei helfen, eine nicht-heteronormative Stadt zu gestalten.

Trotz aller Vorteile, queere Belange und Raumansprüche in die Stadtplanung einzubinden, muss allerdings beachtet werden, dass ein queer inclusive planning Ansatz auf starke Gegenreaktionen stoßen könnte, was letztlich eine stärkere Ablehnung gegenüber Queers und ihren Bedürfnissen hervorrufen 
könnte. Doan beschreibt, wie Planende in den USA auf die Gründung der Gay and Lesbian in Planning Division (GLPD) reagierten. Neben großem Unverständnis für die Bedürfnisse von Queers werde die Gründung der GLPD als ,Gefahr für die Mehrheitsgesellschaft' angesehen (vgl. Doan 2011 a: 7ff.). Jenseits der zu erwartenden Ablehnung vieler Planender ist ebenso damit $\mathrm{zu}$ rechnen, dass Bestrebungen um einen queer inclusive planning Ansatz zu einer wirtschaftlich orientierten Instrumentalisierung von Queers führen könnten. Der US-amerikanische Ökonom Richard Florida geht davon aus, dass tolerante Städte eine starke Anziehungskraft auf die Kreativwirtschaft hätten. Hierbei sieht er den Anteil von Lesben und Schwulen als einen Indikator für die Toleranz einer Stadt (vgl. Berlin Institut für Bevölkerung und Entwicklung 2007: 5 ff.). Die Gefahr hierbei ist, dass Städte einen queer inclusive planning Ansatz nur deshalb verfolgen könnten, um einen wirtschaftlichen Aufschwung zu erreichen. Dies ist bedenklich, weil Queers im Sinne einer positiven Diskriminierung als Wirtschaftsfaktor einer Stadt behandelt werden würden.

Gleichwohl darf nicht erwartet werden, dass allein die Umsetzung eines queer inclusive planning Ansatzes zu einem Umdenken in der Gesellschaft und zu mehr Akzeptanz gegenüber normabweichenden Lebensformen führt. Die veränderten gesetzlichen Rahmenbedingungen für einige Queers vor allem für lesbische und schwule Europäer_innen - sowie die gestiegene Akzeptanz in den letzten Dekaden zeugen davon, dass die politischen Kämpfe von Queers etwas bewirken. Christopher Street Day Paraden (CSD) und insbesondere Dyke-Märsche sowie der Transgeniale CSD in Berlin zeigen, wie Queers selbst ihren Anspruch auf Raum artikulieren und eine temporäre Dekonstruktion heteronormativer Ordnung herbeiführen (vgl. Schuster 2010: 80f.). Ebenso bestätigt das Beispiel des Berliner Stadtteils Schöneberg, dass sich Queers trotz aller Repressalien dauerhaft Raum aneignen können. Gleichwohl trägt die Machtposition deutscher schwuler Männer in Berlin-Schöneberg dazu bei, dass die Belange anderer Queers ausgeklammert werden (vgl. Çetin 2015: 40).

Obgleich diese Formen der Raumaneignung illustrieren, wie Queers selbst dazu beitragen, ihre Belange durchzusetzen, kann die Stadtplanung hierbei unterstützend wirken. Das ,gezielte Einwirken auf die räumliche Entwicklung der Gesellschaft“ (Turowski 1995: 775) ermöglicht der Stadtplanung, an der Dekonstruktion heteronormativer Denkmuster mitzuwirken und Belange von Queers in die Stadtplanung einzubinden. Durch die Auseinandersetzung mit queeren Raumansprüchen hat die Stadtplanung weiterhin die Möglichkeit, ihre exkludierenden Strukturen zu reflektieren und einen wertvollen Beitrag für eine räumliche Inklusion zu leisten.

\section{Autor_innen}

Rogério de Jesus Pereira Lopes forscht zu der Rolle von Queers in der Stadtplanung. rogerio.lopes@tu-dortmund.de 


\section{Literatur}

Andersson, Johan (2012): Heritage discourse and the desexualisation of public space: the "historical restorations" of Bloomsbury's Square. In: Antipode 44/4, 1081-1098.

Associated Press (2015): New York City makes Stonewall Inn a landmark. http://www. nytimes.com/2015/o6/24/nyregion/new-york-city-makes-stonewall-inn-a-landmark. html?_r=o (letzter Zugriff am 4.7.2016).

Berlin-Institut für Bevölkerung und Entwicklung (2007): Talente, Technologie und Toleranz - wo Deutschland Zukunft hat. Berlin: Berlin-Institut.

Castells, Manuel (1983): The City and the Grassroots - a Crosscultural Theory of Social Movements. Berkeley/Los Angeles: University of California Press.

Çetin, Zülfukar (2015): Der Schwulenkiez - Homonationalismus und Dominanzgesellschaft. In: Iman Attia / Swantje Köbsell / Nivedita Prasad (Hg.), Dominanzkultur reloaded. Neue Texte zu gesellschaftlichen Machtverhältnissen und ihren Wechselwirkungen. Bielefeld: Transcript Verlag, 35-46.

Czollek, Leah Carola / Perko, Gudrun / Weinbach, Heike (2009): Lehrbuch Gender und Queer: Grundlagen, Methoden und Praxisfelder. Weinheim: Juventa Verlag.

Diebäcker, Marc (2008): Governance und Demokratie. In: Alex Hamedinger / Oliver Frey / Jens S. Dangschat / Andrea Breitfuss (Hg.), Strategieorientierte Planung im kooperativen Staat. Wiesbaden: VS Verlag, 266-281.

Doan, Petra L. (2007): Queers in the american city: transgendered perceptions of urban space. In: Gender, Place and Culture 14/1, 57-74.

Doan, Petra L. (2011a): Why question planning assumptions and practices about queer spaces. In: Doan, Petra L. (Hg.), Queerying Planning - Challenging Heteronormative Assumptions and Reframing Planning Practice. Farnham / Burlington: Ashgate, 1-20.

Doan, Petra L. (2011b): Queerying identity - planning and the tyranny of gender. In: Petra L. Doan (Hg.), Queerying Planning - Challenging Heteronormative Assumptions and Reframing Planning Practice. Franham/Burlington: Ashgate, 89-128.

Doan, Petra L. (2011c): Conclusion and reflections for the future - refraiming planning practices. In: Petra L. Doan (Hg.), Queerying Planning - Challenging Heteronormative Assumptions and Reframing Planning Practice. Farnham/Burlington: Ashgate, 221-230.

Doan, Petra L. / Higgins, Harrison (2011): The demise of queer space? Resurgent gentrification and the assimilation of LGBT neighborhoods. In: Journal of Planning Education and Research, Jg. 2011, 31/1, 6-25.

Doan, Petra L. (2015): Why plan for the LGBTQ community?. In: Petra L. Doan (Hg.), Planning and LGBTQ Communities - The Need for Inclusive Queer Spaces. New York: Routledge, 1-15.

Dubrow, Gail (2011): Lavender landmarks revisited - advancing an LGBT preservation agenda. In: Petra L. Doan (Hg.), Queerying Planning - Challenging Heteronormative Assumptions and Reframing Planning Practice. Farnham/ Burlington: Ashgate, 53-70.

Dworek, Günter (2012): § 175 StGB: „weggefallen“ - nach 123 Jahren. In: Hirschfeld Eddy Stiftung (Hg.), Vom Verbot zur Gleichberechtigung - Die Rechtsentwicklung zu Homosexualität und Transsexualität in Deutschland. Berlin: Hirschfeld Eddy Stiftung, 46-57.

Edelman, Lee (2004): No Future: Queer Theory and the Death Drive. Durnham: Duke University Press.

El-Tayeb, Fatima (2015): Anders Europäisch: Rassismus, Identität und Widerstand im vereinten Europa. Münster: Unrast.

Engel, Antke (1994): Abschied von der Binarität? Die Kategorie Geschlecht im feministischphilosophischen Diskurs seit Mitte der 8oer Jahre. Hamburg: Universität Hamburg.

Fainstein, Susan S. / Fainstein, Norman (1996): City planning and political values - an updated view. In: Scott Campbell / Susan S. Fainstein (Hg.), Readings in Planning Theory. Oxford: Blackwell, 256-266.

Fincher, Ruth / Iveson, Kurt (2008): Planning and Diversity in the City. Basingstoke: Palgrave Macmillan.

Forsyth, Ann (2001): Sexuality and space: nonconformist populations and planning practice. In: Journal of Planning Literature 15/3, 339-558.

Forsyth, Ann (2011): Queerying planning practice - understanding non-conformist populations. In: Petra L. Doan (Hg.), Queerying Planning - Challenging Heteronormative Assumptions and Reframing Planning Practice. Farnham/Burlington: Ashgate, 21-52.

Forsyth, Ann / Cheung, George (2001): Queers and planning. http://www.plannersnetwork. org/2001/o3/queers-and-planning/ (letzter Zugriff am 4.7.2016). 
Frisch, Michael (2002): Planning as a heterosexist project. In: Journal of Planning Education and Research 21/3, 254-266.

Horchler, Andreas (2016): Auf breiter Front gegen Homo-Ehe. https://www.tagesschau.de/ ausland/homosexuelle-usa-suedstaaten-101.html (letzter Zugriff am 14.5.2016).

Hubbard, Phil (2012): Cities and Sexualities. Milton Park: Routledge.

Huning, Sandra (2014): Wer plant für wen? Partizipation im Kontext gesellschaftlicher Differenzierung. In: Patrick Küpper / Meike Levin-Keitel / Friederike Maus / Peter Müller / Sara Reimann / Martin Sondermann / Katja Stock / Timm Wiegand (Hg.), Raumentwicklung 3.0 - Gemeinsam die Zukunft der räumlichen Planung gestalten. Arbeitsbericht der ARL 8. Hannover: Akademie für Raumforschung und Landesplanung.

Jagose, Annamarie (1996): Queer Theory - Eine Einführung. Übersetzung: Corinna Genschel / Caren Lay / Nancy Wagenknecht / Volker Woltersdorff. Berlin: Querverlag.

de Jesus Pereira Lopes, Rogério (2015): Queer Planning. Die Rolle von Queers in der Stadtplanung. Technische Universität Dortmund: unveröffentlichte Bachelorarbeit.

Klapeer, Christine M. (2015): Vielfalt ist nicht genug! Heteronormativität als herrschaftsund machtkritisches Konzept zur Intervention in gesellschaftliche Ungleichheiten. In: Friederike Schmidt / Anne-Christin Schondelmayer / Ute B. Schröder (Hg.): Selbstbestimmung und Anerkennung sexueller und geschlechtlicher Vielfalt - Lebenswirklichkeiten, Forschungsergebnisse und Bildungsbaustein. Wiesbaden: Springer VS, 25-44.

Koordinierungsstelle u. Stadtplanungsamt 9.7.2015: Koordinierungsbeauftragte_rder Stadt Dortmund für Lesben, Schwule und Transidente u. Stadtplaner_in der Stadt Dortmund. Interview im Stadtplanungsamt Dortmund.

Kühn, Manfred (2008): Strategische Stadt- und Regionalplanung. In: Raumforschung und Raumordnung 66/3, 230-243.

Lee, John A. (1990): Cruising. In: Wayne R. Dynes (Hg.), The Enyclopedia of Homosexuality. New York: Garland Publishing, 284-285.

van Liehout, Maurice (1997): Leather nights in the woods - locating male homosexuality and sadomasochism in a dutch highway rest area. In: Anne Marie Bouthillette / Yolanda Retter / Gordon Brent Ingram (Hg.), Queers in Space - Communities, Public Spaces, Sites of Resistance. Seattle: Bay Press, 339-356.

National Coalition of Anti-Violence Programs (2015): Lesbian, Gay, Bisexual, Transgender, Queer, and HIV-affected Hate Violence in 2014. 2015 Release Edition. New York: National Coalition of Anti-Violence Programs.

National Park Service (2016): Civil rights at Stonewall national historic landmark. https:// www.nps.gov/places/stonewall.htm (letzter Zugriff am 4.7.2016).

Scally, Derek (2015): Gauck fordert offene Debatte über Ehe für alle. http://www.zeit. de/gesellschaft/zeitgeschehen/2015-07/gauck-irland-homo-ehe (letzter Zugriff am 14.5.2016).

Schulz, André (2009): Strategisches Diversitätsmanagement: Unternehmensführung im Zeitalter der kulturellen Vielfalt. Wiesbaden: Springer Gabler.

Schuster, Nina (2010): Andere Räume - Soziale Praktiken der Raumproduktion von Drag Kings und Transgender. Bielefeld: Transcript Verlag.

Stock, Marion (2008): Stolpersteine, Störungen, Probleme: Bürgerbeteiligung in der Stadtentwicklung als Herausforderung für das Prozessmanagement. In: Newsletter Wegweiser Bürgergesellschaft 12/2008 vom 20.6.2008, 1-9.

de Tomás, Alfonso (2016): US-Justizminister verklagt North Carolina. http://www.lto.de/ recht/nachrichten/n/transgender-gesetz-north-carolina-klage-usa-justizministeriumdiskriminierung/ (letzter Zugriff am 14.5.2016).

Turowski, Gerd 1995: Raumplanung. In: Akademie für Raumforschung und Landesplanung (Hg.), Handwörterbuch der Raumordnung. Hannover: Akademie für Raumforschung und Landesplanung, 774-776.

Wagenknecht, Peter (2007): Was ist Heteronormativität? Zu Geschichte und Gehalt des Begriffs. In: Jutta Hartmann / Christian Klesse / Peter Wagenknecht / Bettina Fritzsche / Kristina Hackmann (Hg.), Heteronormativität: Empirische Studien zu Geschlecht, Sexualität und Macht. Wiesbaden: Springer Fachmedien, 17-34.

Wortmann, Sabine (2008): Lebensstilkonflikte sozialer Minderheiten im Berliner Quartiersmanagement Schöneberger Norden. Humboldt-Universität zu Berlin: Dissertation.

Young, Adam (2016): Seattle Parks \& Recreation: All Gender Restrooms / Changing Room Study. Final Report. Seattle: Young Architecture LLC. 
